\title{
Suivre les évolutions du travail et de la santé : EVREST, un dispositif commun pour des usages diversifiés
}

Monitoring changes in work and health: EVREST, a common tool for diverse uses Seguimiento de la evolución del trabajo y de la salud: EVREST, un dispositivo común para usos diversificados

\section{Anne-Françoise Molinié et Ariane Leroyer}

\section{(2) OpenEdition}

\section{Journals}

\section{Édition électronique}

URL : http://journals.openedition.org/pistes/1852

DOI : 10.4000/pistes. 1852

ISSN : 1481-9384

Éditeur

Les Amis de PISTES

\section{Édition imprimée}

Date de publication : 1 novembre 2011

\section{Référence électronique}

Anne-Françoise Molinié et Ariane Leroyer, « Suivre les évolutions du travail et de la santé : EVREST, un dispositif commun pour des usages diversifiés ", Perspectives interdisciplinaires sur le travail et la santé [En ligne], 13-2 | 2011, mis en ligne le 01 novembre 2011, consulté le 30 avril 2019. URL : http:// journals.openedition.org/pistes/1852; DOI : 10.4000/pistes.1852

Ce document a été généré automatiquement le 30 avril 2019.

\section{cc) (†)}

Pistes est mis à disposition selon les termes de la licence Creative Commons Attribution - Pas d'Utilisation Commerciale - Pas de Modification 4.0 International. 


\section{Suivre les évolutions du travail et de la santé : EVREST, un dispositif commun pour des usages diversifiés}

Monitoring changes in work and health: EVREST, a common tool for diverse uses Seguimiento de la evolución del trabajo y de la salud: EVREST, un dispositivo común para usos diversificados

\section{Anne-Françoise Molinié et Ariane Leroyer}

1 Le dispositif Evrest (ÉVolutions et RElations en Santé au Travail) est un observatoire pluriannuel par questionnaire, construit en collaboration par des médecins du travail et des chercheurs, pour pouvoir analyser et suivre différents aspects du travail et de la santé de salariés.

2 Le recueil des données s'appuie sur un questionnaire très court, qui tient sur un rectoverso, rempli lors des consultations (voir questionnaire en annexe). Ce questionnaire a été pensé pour être proche de la conduite habituelle de l'entretien de santé au travail, afin de s'intégrer au mieux dans la consultation du médecin du travail. Les questions portent sur les conditions de travail, la formation, le mode de vie et l'état de santé lors de l'entretien. La façon d'aborder le travail est à la fois large par la diversité des domaines évoqués (changement de travail en lien éventuel avec la santé, horaires de travail et leur régularité, contraintes de temps et pression temporelle, sens et vécu du travail, charge physique, exposition à certains risques, formation reçue ou dispensée) et très succincte compte tenu du faible nombre de questions dans chacun de ces champs. Le libellé des questions sur le travail reprend souvent celui utilisé dans des enquêtes nationales faisant référence.

Ce dispositif vise d'une part à constituer une base nationale en vue de travaux scientifiques, à partir d'un échantillon de salariés vus par les médecins du travail volontaires pour participer à Evrest, d'autre part à permettre à chaque médecin participant (ou à un groupe de médecins qui décideraient de se coordonner) de produire 
et d'exploiter ses propres données pour nourrir les réflexions sur le travail et la santé dans une collectivité de travail. Il s'agit donc d'un dispositif commun à des acteurs relevant de mondes professionnels différents et qui s'inscrit dans un espace d'échanges entre eux, mais aussi que chacun des acteurs s'approprie (voire adapte) à l'intérieur de son activité propre et de ses objectifs.

4 L'intérêt du dispositif Evrest, son apport aux connaissances et aux débats sur les évolutions du travail et de la santé des salariés, mais aussi les difficultés auxquelles il est confronté, doivent être analysés en fonction des enjeux liés à ses particularités comme une opération de coconstruction et de suivi d'informations sur le travail et la santé des salariés, mais aussi comme un dispositif articulant la production de données nationales et un usage « de terrain » par des médecins du travail. La tension entre la structure partagée qui permet le travail commun et les usages "locaux» est une dimension fondatrice d'Evrest. Ces caractéristiques nous ont paru assez proches de celles retenues par Susan Leigh Star et James R. Griesemer (Star et (la perluète ne peut s'utiliser que dans les noms de raison sociale) Griesemer, 1989) quand ils ont proposé la notion d'« objet-frontière » pour analyser les mécanismes de coordination du travail scientifique dans une situation de coopération entre des acteurs relevant de " communautés de pratiques » différentes.

Nous commencerons donc par présenter rapidement cette notion et en quoi elle nous semble pertinente pour comprendre le dispositif Evrest. Dans une seconde partie nous reviendrons sur les convictions partagées par les acteurs relevant de mondes professionnels différents, partage sans lequel la coopération autour du projet Evrest n'aurait pas été possible. Ceci nous conduira, dans une troisième partie, à présenter les « objets » qui structurent l'espace d'échanges. Enfin, nous terminerons par l'analyse des défis et tensions qui constituent la trame sur laquelle devra se tisser la pérennité du dispositif.

Notre participation au dispositif nous permet de connaître finement la longue histoire de sa construction et des multiples négociations qui sont intervenues au cours de ce processus. Mais elle rend difficile la prise de distance qui serait nécessaire pour une analyse de la diversité des pratiques liées à l'émergence de cette « infrastructure ». Nous sommes toutes deux fortement impliquées dans le dispositif national Evrest, au sein de l'équipe projet nationale. L'une de nous a une longue expérience de la quantification dans le champ du travail et de la santé et de réflexion sur la place de cette démarche en lien avec les préoccupations de l'ergonomie de l'activité (Molinié et Volkoff, 1982; Molinié, 2003 ; Volkoff et Molinié, 2010). En collaboration avec S. Volkoff, elle a participé à l'émergence du dispositif au sein d'une grande entreprise aéronautique française et a joué par la suite un rôle de "passeur » entre cette expérience et les réflexions d'un groupe de médecins de services interentreprises, réunis au sein d'un groupe "épidémiologie ». L'autre, plus ancrée initialement dans la recherche en épidémiologie et en santé publique, a rencontré Evrest lors de la mise en place de la phase d'expérimentation du dispositif national dans une région française. Elle a été très fortement impliquée dans l'extension de ce dispositif, les outils dont il s'est doté, les débats auxquels il a donné lieu, les résultats qu'il a permis de produire à différents niveaux, etc. Elle assure actuellement la direction du groupement d'intérêt scientifique qui s'est constitué autour d'Evrest. 


\section{Un dispositif issu des échanges entre chercheurs et médecins du travail}

\subsection{La notion d'« objet-frontière »}

7 Susan Leigh Star et James R. Griesemer (op. cit.) ont forgé la notion d'« objet-frontière » pour décrire et caractériser les divers processus par lesquels des acteurs relevant de mondes sociaux différents, mais appelés à coopérer sur un projet scientifique, réussissent à se coordonner malgré leurs points de vue différents; dans ce processus chacune des parties garde son identité, ses enjeux et peut mener ses travaux, tout en s'articulant avec les autres. Dans cet article originel, il s'agit d'une coopération durable (1907-1939) entre des acteurs extrêmement divers (chercheurs, administratifs, gestionnaires, naturalistes amateurs, taxidermistes, fondations, politiques) autour de la construction d'un musée de zoologie au sein de l'Université de Californie. Les solutions inventées par les acteurs en situation sont de deux ordres: une relative standardisation des méthodes (non en fonction des seules exigences scientifiques, mais au terme d'un processus de « réconciliation » entre les intérêts des différents acteurs) et la mise au point d'objetsfrontière. La notion d'objet-frontière est
«an analytic concept of those scientific objects which both inhabit several intersecting social worlds and satisfy the informational requirements of each of them. Boundary objects are objects which are both plastic enough to adapt to local needs and the constraints of the several parties employing them, yet robust enough to maintain a common identity across sites. [...] They have different meanings in different social worlds but their structure is common enough to more than one world to make them recognizable, a means of translation. The creation and management of boundary objects is a key process in developing and maintaining coherence across intersecting social worlds. » (Op. cit., p. 393).

Avec la notion d'objet-frontière, il s'agit « d'entrer dans la mécanique des associations elle-même et la façon dont elles supportent la tension entre coopération et hétérogénéité » (Trompette et Vinck, 2009, p.9). Dans cette perspective, le terme « frontière » est moins perçu comme renvoyant à une limite de territoire que comme

"un espace partagé, le lieu précis où le sens de l'ici et du là-bas se rejoignent. Ces
objets communs constituent des frontières entre groupes grâce à la flexibilité et à la
structure partagée; ils sont des ingrédients de l'action. » (Star, 2010, p. 20).

Quant aux « objets», ils peuvent être matériels ou immatériels; ils sont définis non par leurs caractéristiques intrinsèques, mais en référence à l'action et à la coopération ${ }^{1}$. C'est parce qu'il s'agit de faire des choses ensemble, d'œuvrer à un projet commun, que ces objets sont construits dans la rencontre d'acteurs relevant de communautés de pratiques différentes. Ils sont pertinents pour l'action dans le cadre du projet commun, mais ils sont aussi mobilisés à des fins qui sont propres à chacun des acteurs, dans leurs pratiques respectives qui, elles, ne sont pas interdisciplinaires.

10 Parmi les propriétés des objets-frontière, la «flexibilité interprétative " a été particulièrement investie, un "même » objet pouvant avoir un sens différent selon l'utilisation et l'interprétation qu'en font ceux qui s'y réfèrent. Cependant, certaines dimensions de la notion originale sont souvent oubliées, et notamment celle 
«de l'infrastructure invisible auxquelles les objets-frontière contribuent par les conventions et les standards qu'ils véhiculent » (Trompette et Vinck, 2010, p. 11) (voir aussi Bowker et Star, 1999).

11 Si nous avons retenu cette notion pour réfléchir au dispositif Evrest, c'est parce qu'elle permet de penser de façon indissociable les formes de la construction commune (objets et infrastructure) et la tension - inhérente au dispositif - entre la perspective d'un projet commun et l'hétérogénéité des acteurs et des pratiques, et de saisir les "intersections » par-delà l'exigence de consensus. Dans le présent article, nous mettrons surtout l'accent sur la dynamique de la coopération entre chercheurs et médecins du travail et la façon dont elle a façonné le mode de construction et les formes du dispositif. Notre propre positionnement, mais aussi la jeunesse du dispositif, ne nous autorisent pas à développer une analyse fine des usages d'Evrest dans les pratiques des médecins du travail au sein des entreprises et des effets de la quantification sur le champ de la santé au travail en entreprises. Mais Evrest fait par ailleurs l'objet d'un volet d'une thèse de sociologie en cours $^{2}$ sur les pratiques et les effets sociaux de la quantification dans le champ de la santé au travail, au sein de grandes entreprises. Une partie des travaux conduits dans ce contexte ont contribué à alimenter notre propre réflexion sur Evrest.

\subsection{Le creuset de la naissance d'Evrest}

12 Le contexte de la naissance d'Evrest dans une grande entreprise aéronautique (Gilles, 2006; Gilles et Volkoff, 2009) a contribué à lui donner certaines caractéristiques. La genèse d'Evrest dans cette entreprise est très liée aux préoccupations portées par une femme médecin du travail et ergonome, à l'origine de la création (en 1985) d'un département d'ergonomie, puis à la tête du département des facteurs humains au sein de la direction générale. L'entreprise était alors confrontée à des difficultés après la mise en œuvre de mesures d'âge, qui ont révélé des enjeux liés à des risques de perte de compétences essentielles, dans un domaine de très haute technologie où la fiabilité est un impératif. Toute une démarche a alors été mise en place visant, entre autres, à construire des repères pour que les responsables de production et des ressources humaines puissent prendre en compte les savoirs et les savoir-faire des opérateurs (Doppler 1995). Cette démarche a aussi constitué une occasion pour que l'ergonomie pénètre dans l'univers de la conception et pour tenter de sortir les enjeux de santé d'une vision étroite de la prévention (" loin de se cantonner à l'éviction du risque de pathologies, elle cherche à favoriser la santé vue comme un processus de développement " [Doppler, 2004]). Elle a été conduite en s'appuyant sur des relations étroites et des coopérations avec des chercheurs dans le champ de l'ergonomie, chercheurs avec lesquels des liens s'étaient créés lors de la formation en ergonomie de cette responsable de l'entreprise (Falzon et Doppler, 1998).

Des éléments permettant de réfléchir aux relations entre changements techniques et organisationnels et caractéristiques du travail et de la population (en particulier d'âge et d'ancienneté) ont alors été introduits à différentes étapes de plusieurs projets de conception ou d'aménagement. Il s'est agi aussi de montrer que l'analyse de l'activité peut enrichir les démarches de conception, en fournissant des éléments sur la variabilité des situations réelles de travail. Ces préoccupations ont été intégrées à la formation des concepteurs, et complétées par des repères sur les caractéristiques du fonctionnement humain. L'accent a été mis aussi sur la nécessité d'une analyse intégrant la dimension temporelle des phénomènes, et en particulier l'importance 
«de prendre en compte et d'expliciter l'expérience professionnelle passée [des opérateurs], leur savoir-faire. Il faut pouvoir intégrer le passé pour se projeter dans l'avenir. [...]. La dimension historique est une caractéristique essentielle de la dynamique du fonctionnement humain qui est bien souvent oubliée dans le monde de l'entreprise ». (Doppler, 1995, p. 422).

14

On retrouvera dans Evrest le critère de l'âge et cette dimension dynamique pour introduire une réflexion plus large sur les relations entre santé et travail.

Enfin, pour pénétrer l'univers des concepteurs, jeter « un pont entre le monde de la technique et celui des $R H$ » (Falzon et Doppler, op. cit.), une réflexion a été amorcée sur la possibilité d'introduire des indicateurs renvoyant à ces préoccupations dans les " tableaux de bord " utilisés habituellement par les décideurs de l'entreprise. Avec peu à peu l'idée que les médecins du travail peuvent y contribuer, en

«développant une approche collective et en apportant aux décideurs, aux partenaires sociaux, des données chiffrées sur les relations santé et travail» (extrait d'un compte rendu de groupe de travail interne).

Du côté des médecins du travail de l'entreprise, la quantification apparaît comme une ressource pour rendre visibles les enjeux de santé dans leurs relations avec le travail, mais aussi leur propre activité et leur utilité. Ils espèrent que ces nouveaux « indicateurs" pourront contribuer à transformer les représentations et ouvrir des espaces nouveaux de mise en débat des questions de santé au travail, espaces dans lesquels leurs points de vue auront mieux droit de cité.

Ces préoccupations vont rencontrer celles de chercheurs du Créapt (Centre de recherches et d'études sur l'âge et les populations au travail), avec qui une collaboration avait été amorcée sur la thématique de l'âge et du travail, autour d'études ergonomiques et démographiques au sein de l'entreprise (Millanvoye et Colombel, 1996) et qui avaient euxmêmes un parcours professionnel marqué par une réflexion et une pratique sur la quantification dans les domaines du travail et de la santé.

Dans cette rencontre s'est forgé un premier dispositif Evrest (Archambault et coll., 2006) dont les grandes caractéristiques persistent aujourd'hui :

- un dispositif construit peu à peu en coopération avec des chercheurs ;

- un dispositif ancré dans la pratique des médecins du travail et porté par eux ;

- un dispositif conçu pour durer - donc forcément plus léger et « généraliste » qu'une enquête ponctuelle sur un problème particulier ;

- un dispositif visant à produire des « indicateurs » à la fois sur le travail et sur la santé, et sur leur évolution ;

- un dispositif qui puisse faire écho aux préoccupations de l'ergonomie de l'activité.

Ce dispositif a été repris et adapté afin de pouvoir être utilisé par les médecins du travail de services interentreprises (donc pour de plus petites entreprises) et d'autres services autonomes.

\subsection{Un dispositif qui va « intéresser » au-delà d'une entreprise}

Le dispositif national Evrest est issu de la rencontre entre les chercheurs qui avaient participé à la création du dispositif dans cette grande entreprise aéronautique et des médecins du travail de services interentreprises. Ces médecins du travail, réunis dans un "groupe Épidémiologie» au sein du Cisme ${ }^{3}$, avaient pour la plupart d'entre eux une certaine appétence pour les approches quantifiées, une formation en épidémiologie, et 
une pratique commune d'enquêtes ponctuelles sur des sujets divers. La rencontre s'est tissée à l'occasion d'une réflexion de ce groupe de médecins sur les enjeux de travail et de santé en fin de vie active. Ils ont souhaité faire appel à des chercheurs, connus à la fois pour leurs travaux dans le champ du vieillissement au travail et pour leur expérience des enquêtes dans ce domaine, en lien avec des médecins du travail (Derriennic, Touranchet et Volkoff, 1992 ; Coutrot et Molinié, 2006; Molinié et Volkoff, 2000). Dans les échanges qui ont eu lieu autour de ce projet, puis lors de la concrétisation avec l'enquête Santé et vie professionnelle après 50 ans (SVP50), de nombreux déplacements se sont opérés au sein de ce groupe: une connexion plus importante avec la sphère de la statistique publique (examen de diverses enquêtes pour repérer les questionnements utilisés) et des débats sociaux nationaux (notamment à travers le Conseil d'orientation des retraites - qui accordera un financement pour l'enquête SVP50); l'émergence de préoccupations liées à l'univers de la recherche (examen de la littérature dans un champ de connaissances, participation à des colloques, publications scientifiques, etc.) ; un approfondissement de la réflexion sur la place de la quantification dans le champ de la santé au travail et dans la pratique clinique des médecins du travail, etc. Leur réflexion sur la quantification a conduit ces médecins à avoir un grand intérêt pour l'expérience de l'observatoire en santé au travail au sein de l'entreprise de construction aéronautique, qui entrait bien en résonance avec des préoccupations qu'ils avaient déjà échangées au sein du groupe, mais avec le souhait de réfléchir à la spécificité de leur pratique et du suivi de salariés travaillant dans des entreprises de plus petite taille et de secteurs diversifiés.

\section{Des préoccupations partagées}

21 Un processus de coconstruction entre des acteurs issus de mondes professionnels différents ne suppose pas forcément d'avoir établi un consensus préalable. Il est cependant nécessaire qu'il existe un certain nombre de préoccupations partagées qui donnent envie de travailler ensemble - et sur la durée - autour d'un projet et que se créent des modalités d'échanges qui permettent à la fois la construction commune et la préservation des spécificités. Nous évoquerons quelques préoccupations partagées qui ont rendu possible la naissance et le développement d'Evrest, même s'il est probable que les pondérations entre ces différentes préoccupations diffèrent selon les personnes participant au projet.

\subsection{L'affirmation d'un intérêt de la quantification pour des réseaux d'acteurs de la santé au travail}

Médecins comme chercheurs, l'usage de la quantification leur semblait présenter plusieurs intérêts dans le contexte des évolutions du travail. Le premier est lié à la persistance, dans les pays industrialisés et en France en particulier, d'un niveau élevé de contraintes et nuisances dans le travail, facteur important d'inégalités sociales de santé et peu prises en compte dans les politiques publiques et les négociations sociales (Arnaudo et coll., 2004 ; Bué et coll., 2007 ; Gollac et Volkoff, 2007). Les chiffres ont l'intérêt de venir renforcer, en les "lestant» du poids de la quantification, une série de constats, qui rappellent par exemple que la "pénibilité " du travail ne relève pas seulement de l'histoire ancienne, ou que les questions de santé ne sont pas indépendantes de ce qui se joue dans le travail. Un second intérêt peut être mis en lien avec la tendance à 
l'intensification du travail, perceptible depuis une vingtaine d'années (Askenazy et coll., 2006). Ses effets sur la santé, souvent indirects, sont très variables d'une personne à l'autre et souvent renvoyés au vécu individuel - ce qui rend difficilement visible le lien entre travail et santé (Volkoff, 2008). La quantification peut être une des voies qui permet de sortir de cette individualisation accrue des liens santé/travail. Enfin, pour révéler ces difficultés, fournir des éléments d'appréciation, et contribuer à ouvrir des possibilités de transformation, les médecins du travail peuvent (ou pourraient) jouer un rôle majeur. Or, la quantification est aussi un «langage carrefour" (Desrosières, 1993) qui peut aider à donner droit de cité à des préoccupations dans des sphères de discussion ou de décision dans lesquelles, trop souvent, ce qui ne se chiffre pas n'existe pas.

Les acteurs concernés partagent aussi la conviction que les indicateurs auxquels on se réfère usuellement en entreprise quand on parle de santé au travail sont d'une faible utilité pour étayer ces préoccupations. En effet, les statistiques d'accidents du travail et de maladies professionnelles constituent le socle essentiel des évaluations statistiques en santé au travail dans l'entreprise; or elles se posent comme des outils de mesure alors qu'elles sont entièrement imbriquées dans les pratiques de gestion des risques et de tarification. C'est un exemple de ce que Desrosières (2004) appelle la « circularité » entre les données permettant d'évaluer un fait social et les instruments choisis pour agir sur son évolution. Quant aux indicateurs issus des rapports annuels fournis par les médecins du travail, ils ciblent plus l'activité mise en œuvre (visites médicales, actes paramédicaux, activités de tiers temps, etc.) que la santé au travail.

D'où l'idée de trouver un dispositif, ancré dans la pratique des médecins du travail, qui favorise la mise en visibilité d'une pluralité de dimensions du travail et de la santé ; qui permette un suivi, pour apprécier des relations de moyen ou long terme; et qui puisse fournir des connaissances utiles à la prévention, ou plus largement à la transformation des situations de travail, tout en s'articulant avec les pratiques de différents acteurs dans ces domaines, et en les renforçant dans leur pouvoir de conviction.

\subsection{Un « modèle » partagé des relations entre le travail et la santé}

Peut-être parce qu'ils avaient été confrontés aux questions d'âge et de parcours professionnels et y avaient travaillé ensemble, les médecins du travail et les chercheurs qui ont conçu le dispositif national Evrest ont choisi d'aborder les enjeux de santé au travail sans préjuger du sens des relations causales entre ces deux termes, et en les inscrivant dans une perspective diachronique. En effet, sans minimiser l'importance - et la grande méconnaissance - des atteintes à la santé qui trouvent leur origine dans les conditions de réalisation du travail, ils ne souhaitaient pas traiter des relations santétravail uniquement dans le registre de l'altération, de la dégradation ou de la fragilisation de la santé par le travail. Une telle orientation aurait laissé dans l'ombre la façon dont la santé joue sur la vie professionnelle et se joue dans la vie professionnelle, et le rôle important du travail dans la construction et le développement de la santé.

L'interrogation sur le travail ne vise donc pas principalement à repérer des " expositions ", même si elle comporte certaines questions sur ce sujet. Cette notion, certes précieuse en épidémiologie, laisse cependant "supposer que les opérateurs sont passifs dans un environnement à risques» (Laville, 1998, p. 154). L'ergonomie montre que les opérateurs agissent sur leurs « conditions de travail », notamment pour préserver ou construire leur santé ; ces stratégies sont plus ou moins possibles, limitées, efficientes, selon les marges 
de manœuvre dont ils disposent. Dans cette perspective, un questionnaire fondé sur les réponses des salariés peut présenter des qualités d'intégration,

« les réponses des opérateurs traduisant à la fois une combinaison de contraintes et

l'espace disponible pour leurs propres stratégies de préservation» (Volkoff, 2005,

p. 29-30).

À travers le questionnaire Evrest, on vise moins à isoler des caractéristiques du travail à l'origine de tel ou tel problème de santé qu'à alimenter une démarche exploratoire, compréhensive, qui essaie de prendre en considération plusieurs dimensions d'une situation, de cerner des configurations de relations entre des aspects du travail et des aspects de la santé. De plus, le caractère longitudinal du dispositif permet d'envisager d'instruire ces relations en prenant en compte non seulement des caractéristiques du travail concomitantes de celles de la santé, mais aussi leurs évolutions respectives.

Au-delà du repérage de certaines formes de "pénibilité » du travail (contraintes d'horaires de travail, pénibilité physique), l'interrogation sur le travail proposée dans Evrest voudrait aussi être attentive à la manière dont les salariés peuvent trouver (ou rechercher) dans le travail des ressources de développement de leur santé. Le travail est abordé aussi sous l'angle de la reconnaissance, de la coopération, de la possibilité de faire un travail de qualité, d'occasions de se former ou de jouer un rôle de formateur. Et le questionnaire permet d'examiner - au moins sommairement - la plupart des dimensions considérées comme constitutives des «facteurs psychosociaux de risques ${ }^{4}$ : les exigences du travail (notamment la pression temporelle); l'autonomie et les marges de manœuvre (notamment: pouvoir choisir la façon de procéder, avoir un travail qui permet d'apprendre) ; les conflits de valeur (avoir les moyens pour faire un travail de qualité; devoir faire des choses que l'on désapprouve [question introduite en 2011]); certains aspects du soutien social et de la reconnaissance au travail (avoir des possibilités suffisantes d'entraide, de coopération; travail reconnu par l'entourage professionnel) ou des exigences émotionnelles (travail en contact avec le public); et enfin l'insécurité de l'emploi et du travail (travailler avec la peur de perdre son emploi [question introduite en 2011]). Avec l'idée qu'il ne s'agit pas seulement de repérer des "risques", mais aussi de s'intéresser aux conditions qui permettent à certains salariés de se sentir plutôt bien dans leur travail, au moins pendant une certaine période, au moins pour tel ou tel aspect de leur travail, et que parfois, les actions utiles peuvent viser à préserver certains éléments des situations de travail, et pas seulement à supprimer des nuisances.

L'approche de la santé qui a été retenue s'est d'emblée écartée d'une entrée par les pathologies, même si la question du travail avec une maladie chronique est sans doute de plus en plus souvent présente dans les entreprises et dans les préoccupations des médecins du travail ${ }^{5}$; cependant, elle mérite probablement une investigation spécifique d'une autre nature. Mais nombre de pathologies conduisent encore à l'exclusion de l'emploi, tandis que les troubles de santé de l'ordre de l'infrapathologique peuvent s'avérer plus compatibles avec un maintien de l'activité professionnelle, sous certaines conditions tout au moins. Pour chaque dimension de la santé (cardiorespiratoire, neuropsychique, ostéoarticulaire, etc.), les indicateurs figurant dans Evrest sont de trois ordres : le premier signale l'existence de signes et symptômes, qu'ils aient été ou non validés par des diagnostics médicaux; le second interroge les gênes éventuelles dans le travail liées à ce trouble de santé ; le troisième, enfin, porte sur les traitements ou soins mis en place. 
La notion de gêne dans le travail liée à un trouble de santé avait déjà été introduite dans l'enquête SVP50, parce qu'il était apparu qu'il ne suffisait pas de s'intéresser à l'état de santé (même perçu par le salarié) pour en déduire les difficultés qu'il peut générer dans la réalisation de l'activité (Molinié, 2006a et b). Ces difficultés renvoient certes à la nature et à la sévérité des troubles de santé, aux limitations fonctionnelles plus ou moins importantes qui peuvent en résulter, mais aussi aux ressources individuelles et collectives qui peuvent être mobilisées pour y faire face, et à des caractéristiques du travail et de son organisation (contraintes temporelles, entraide, marges de manœuvre...). On peut rapprocher cette préoccupation de celles, plus anciennes, qui ont conduit à des évolutions conceptuelles dans le champ du handicap pour sortir du modèle biomédical et proposer des concepts pertinents pour les échanges et les interventions des professionnels (Nagi, 1964 ; Wood, 1975 ; World Health Organization, 1980 ; Minaire, 1992 ; Fougeyrollas, 2002). Plusieurs notions ont été distinguées : ce qui relève de la maladie ou de la déficience ; les limitations fonctionnelles; les restrictions d'activité ; et le « handicap de situation » ou le "désavantage social». La distinction entre limitation fonctionnelle et restriction d'activité (voire handicap de situation) a permis d'ouvrir sur des interrogations relatives aux stratégies de compensation, individuelles ou collectives, aux ressources dont les personnes disposent (ou pourraient disposer) pour faire face à ces limitations, et aux caractéristiques et contraintes de l'environnement. L'introduction de la notion de gêne dans le travail, de la même façon, ouvre probablement un espace de réflexion qui peut " démédicaliser » la question de la santé au travail en permettant d'une part de redonner une place à l'expérience que les personnes ont construite et qui intègre l'expérience de leurs propres déficiences, et d'autre part d'interroger les ressources qui peuvent se trouver ou se construire dans les situations de travail pour limiter les gênes dans le travail. Ces questions sont également intéressantes dans une perspective longitudinale dans la mesure où elles permettent d'évoquer des signes précoces, attirant l'attention sur les possibilités d'éviter certaines dégradations ultérieures de la santé en relation avec le travail et une éventuelle désinsertion professionnelle.

\subsection{Une approche reposant sur les appréciations des salariés}

31 S'agissant de santé au travail, médecins du travail et chercheurs s'accordent sur l'importance de recueillir les appréciations des salariés eux-mêmes. Ce qui a été évoqué précédemment conduit à exclure une interprétation des «données » qui considérerait qu'elles retranscrivent purement et simplement une réalité extérieure aux personnes enquêtées. Il faut toutefois discuter une autre interprétation selon laquelle les données de l'enquête traduiraient une vision purement subjective, voire arbitraire.

Cette question a largement été traitée à propos des réponses données par les salariés lors des enquêtes nationales sur les conditions de travail (Gollac, 1997) ou de l'enquête ESTEV (Santé travail et vieillissement) (Molinié, 2003, op. cit.). Les réponses spontanées des travailleurs peuvent refléter une vision réductrice de leur activité et de leurs conditions de travail si une élucidation approfondie n'est pas menée avec eux (Teiger et Laville, 1991). Par ailleurs tout questionnaire, toute fiche de recueil de données, sont «adressés ", et les réponses dépendent de la représentation que les salariés se font des attentes et objectifs du destinataire ou commanditaire (pouvoirs publics, employeurs, hiérarchie, syndicats...). Ces réponses vont fluctuer aussi selon l'humeur du jour et les évènements récents. À plus long terme, elles sont influencées par des processus sociaux, qui 
déterminent la visibilité des conditions de travail dans un milieu donné (Gollac, op. cit.). Plus problématique, pour certaines analyses en santé au travail: les réponses sur un aspect du travail peuvent dépendre de l'état de santé. Ainsi le mal de dos renforce les appréciations négatives sur le maintien de postures difficiles, bien davantage que sur les efforts à produire (Duquette et coll., 1997; Balogh et coll., 2004). Enfin, les appréciations des salariés peuvent aussi refléter des ignorances, voire des stratégies défensives (Dejours, 1993). Mais sur certaines questions, la perception qu'ont les personnes est aussi en relation directe avec leur activité. Ainsi, comme le rappelle Michel Gollac (op. cit.), il suffit qu'un travailleur se croie contraint de répondre à la demande, ou se sente obligé de se dépêcher pour que ceci ait un effet réel sur son rythme de travail.

Le libellé des questions d'Evrest reprend souvent celui utilisé dans des enquêtes nationales dont la qualité est reconnue, ce qui permet de bénéficier de ce capital de réflexion sur le sens et l'usage de ces questions, d'une reconnaissance de leur pertinence, et d'ouvrir des possibilités de comparaisons externes - même s'il est sûr que les réponses à une question ne dépendent pas seulement de la formulation de la question, mais aussi du cadre de l'enquête dans laquelle elle s'insère, de ceux qui la portent et de ses objectifs.

Dans le cadre d'Evrest, la place de la parole du salarié est d'autant plus importante que c'est le médecin qui la recueille, et que c'est dans l'échange qui peut se développer à partir d'Evrest que se construit aussi le sens des « données ». Si, le plus souvent, le salarié remplit seul la partie consacrée au travail (le questionnaire lui étant proposé avant l'entrée dans le cabinet médical), ses réponses donnent ensuite lieu à discussion avec le médecin, notamment quand des réponses lui paraissent surprenantes. Cette situation n'est pas exceptionnelle et le médecin du travail peut s'en saisir pour accéder à une meilleure compréhension des enjeux de santé au travail, processus que ne permettrait pas un recueil par un enquêteur extérieur. Evrest peut aussi faciliter l'échange avec certains salariés qui s'expriment peu habituellement, en sollicitant différemment la parole des silencieux, des discrets qui n'osent pas ou ne savent pas dire ce qu'ils vivent ou ce qu'ils ressentent au travail. La participation du médecin à la construction de l'information chiffrée, la qualité de son écoute, l'ensemble des éléments qu'il utilise pour se construire un " point de vue » sur les enjeux de santé au travail dans ce contexte précis sont aussi ce qui lui permettra de porter les résultats dans le débat, en les nourrissant et en les confrontant avec d'autres approches et d'autres points de vue. Le choix d'un questionnaire très court est aussi cohérent avec ce souci de ne pas conférer aux indicateurs chiffrés l'autorité de la preuve et de les insérer dans une démarche plus large de compréhension, intégrant des éléments issus de la clinique médicale.

\section{Une « infrastructure » commune pour des usages diversifiés}

Evrest est, on l'a dit, un dispositif né de la collaboration entre des acteurs de professions différentes, volontaires pour y participer. Sa construction s'inscrit dans un processus visant à rendre compatibles des exigences propres à chacun de ces «mondes" professionnels. Elle passe par la création et le partage d'«objets», partage dans lequel chacun "retrouve son compte", mais aussi par l'élaboration progressive de formes nouvelles de coordination et d'échanges. Le dispositif tient par le fait que ceux qui y participent y trouvent et continueront à y trouver un intérêt, même si la création d'une « infrastructure » cherche à assurer une relative pérennité et une stabilité au dispositif. 
Nous présenterons maintenant les grandes caractéristiques du dispositif, à la fois comme le cadre formel qui a été construit par et pour ces collaborations, mais aussi comme celui qui peut matérialiser différentes façons d'utiliser ces objets, différentes façons pour les acteurs d'être attachés à ces objets. Nous examinerons dans cette perspective plusieurs aspects d'Evrest: le questionnaire (dont le contenu a déjà été décrit précédemment) et l'échantillonnage; le dispositif «technique»; le dispositif «organisationnel»- en essayant à chaque fois de montrer en quoi ils visent à créer une "infrastructure " commune tout en ouvrant la possibilité d'usages diversifiés. C'est-à-dire finalement, ce qui fait qu'on peut les considérer comme des « objets-frontière »...

\subsection{Le questionnaire Evrest : un cadre commun et des questions «libres"}

Le questionnaire Evrest est la clé de voûte du dispositif. Support de l'alimentation de la base de données utilisable pour des travaux de nature scientifique, il constitue aussi une trame d'échanges entre le médecin du travail et les salariés, et l'un des appuis des médecins du travail, seuls ou à plusieurs, pour dresser un "paysage » des conditions de travail dans une entreprise ou une situation professionnelle, et mettre en discussion les enjeux de santé au travail dans ce contexte. C'est aussi un objet partagé qui peut permettre des échanges, entre personnes impliquées dans le réseau Evrest à différents niveaux, et entre ce réseau et d'autres interlocuteurs intéressés par ces questions, tant dans le champ scientifique que dans celui de l'intervention publique ou privée, sur le plan national, régional ou local. Ces multiples facettes ont conduit à réfléchir bien sûr au contenu du questionnaire et à sa longueur, à son mode de passation, mais aussi à sa pertinence et ses limites pour une investigation de terrain, et à sa pertinence scientifique (voir plus haut 2.2 et 2.3) ainsi qu'à la façon de tenir compte des enjeux relevant de l'ensemble de ces dimensions. Il doit aussi pouvoir rester relativement stable pour autoriser des analyses diachroniques, voire longitudinales.

Mais le dispositif est aussi conçu pour permettre des usages spécifiques d'Evrest en fonction des besoins de médecins. Afin d'inclure des questions plus spécifiques et pertinentes pour une population particulière ou sur un sujet particulier, le questionnaire Evrest comporte des zones «libres»; leur existence est prévue dans le questionnaire et ouvre l'accès à une zone de saisie dans la base de données, mais leur contenu est défini par ceux qui les remplissent. Seuls les médecins qui décident d'utiliser ces zones peuvent savoir à quoi correspond ce qui y a été saisi et exploiter les informations correspondantes. À partir de 2011, la part de ces zones «libres» a été fortement augmentée dans le questionnaire, avec deux zones en début de questionnaire visant à repérer par exemple des ateliers ou des métiers spécifiques, une zone permettant de saisir une « exposition » non répertoriée a priori, une question « libre » sur chaque item de santé, et surtout, l'ajout de dix questions totalement « libres » en fin de questionnaire.

Cette évolution importante signe bien le projet d'Evrest, qui ne veut pas être un dispositif national uniformisant et qui « utiliserait » les médecins du travail comme des enquêteurs très qualifiés, mais un dispositif partagé que les médecins peuvent largement faire "à leur main", en fonction de leurs préoccupations locales - mais sans omettre de contribuer à la faisabilité des analyses nationales. On ne sait pas encore quel usage sera fait de ces zones «libres», qui ne prennent de l'ampleur qu'avec les modifications introduites en 2011. Les premières investigations sur ce sujet semblent indiquer qu'elles 
sont surtout utilisées par des réseaux de médecins qui se mettent d'accord pour avoir le même protocole de quelques questions (sur les dix questions «libres ») au sein d'une grande entreprise, ou d'une région, soit pour une enquête ponctuelle (sur une année ou quelques mois), soit avec une perspective d'investigation coordonnée plus durable.

\subsection{L'échantillonnage: les « octobre des années paires » et les autres}

Ces formes d'adaptation du questionnaire à leurs besoins sont en général combinées avec des choix d'élargissement de l'échantillonnage par les médecins. Tout médecin du travail volontaire pour participer à Evrest s'engage à interroger (au moins) tous les salariés nés en octobre des années paires qu'il voit en visite systématique, ce qui constitue une charge de travail qui n'est pas trop forte. Ces salariés constituent l'échantillon national, qui donne lieu aux exploitations nationales et régionales et sur lequel s'appuient les travaux scientifiques dont les chercheurs ont la responsabilité. Mais chaque médecin peut aussi s'il le souhaite élargir son utilisation du dispositif, seul ou avec des confrères, afin de recueillir des données de façon plus systématique pour une entreprise, un secteur professionnel, un métier... Il peut alors adapter l'échantillonnage en fonction du niveau qu'il souhaite atteindre, jusqu'à prendre par exemple l'ensemble des salariés d'une même entreprise.

Il n'est pas encore possible de faire un bilan de ces usages particuliers, qui ne donnent pas lieu à une centralisation systématique, mais peuvent être connus lorsque le médecin référent de la région en a été informé, voire lorsque l'appui d'un statisticien du réseau régional a été sollicité . On a ainsi pu constater que les médecins s'insèrent souvent graduellement dans le dispositif : ils commencent en interrogeant uniquement les salariés nés en octobre des années paires, ce qui permet de se familiariser avec le questionnaire, le dispositif de saisie, et de commencer à s'organiser au sein du service de santé au travail pour intégrer Evrest dans l'activité quotidienne (repérage des salariés nés en octobre des années paires, préparation du questionnaire, organisation de la passation, articulation avec le dossier papier, saisie, etc.). Par la suite seulement, certains médecins s'engagent dans une utilisation plus spécifique d'Evrest dans une entreprise, ou un secteur d'activité. Ceci concernait, à notre connaissance, environ 200 médecins du travail à la fin de 2010. Le tableau au paragraphe 3.3 montre bien l'augmentation progressive du nombre et de la part des fiches "hors échantillon national ", liés à ces usages spécifiques, au fur et à mesure que le dispositif se développe.

\subsection{Les aspects techniques : permettre les exploitations nationales mais aussi donner à chaque médecin des possibilités d'exploiter ses données}

41 Le dispositif technique a donc été conçu à la fois pour des usages scientifiques à partir d'exploitations de l'échantillon national et pour ces usages diversifiés par les médecins utilisateurs. Cela se traduit par différentes possibilités d'accès et de récupération des données: les chercheurs de l'équipe projet nationale ont un accès à l'ensemble des données de l'échantillon national ${ }^{7}$; les médecins référents régionaux accèdent à ces mêmes données, mais seulement pour leur propre région. Quant aux médecins participants, chacun d'eux a accès, via Internet, à un site sécurisé sur lequel il peut saisir 
mais aussi récupérer l'ensemble des données qu'il a saisies (incluses ou non dans l'échantillon national). Toute opération conduite à un autre niveau (un service, un secteur, un métier, etc.) suppose la mise en commun par les médecins concernés (ou avec l'accord explicite de chacun d'eux) de leurs données respectives dans ce champ d'étude.

Un site public d'information (http://evrest.istnf.fr), distinct du site de saisie, met à disposition les outils nécessaires au fonctionnement du dispositif (questionnaire, guide de remplissage, nomenclatures, protocoles, etc.). Mais il fournit également à chaque médecin un programme automatisant la production de résultats simples, sous la forme d'un document reprenant le recto-verso du questionnaire avec, pour chaque question, les résultats concernant la population enquêtée par ce médecin. Le médecin peut ainsi avoir à disposition, aussi souvent qu'il le souhaite, des résultats simples pour l'ensemble des salariés qu'il a interrogés ou pour un sous-ensemble qu'il voudrait sélectionner (une entreprise par exemple). Il peut aussi trouver sur le site divers outils (diaporamas modulables) facilitant la présentation de résultats.

Le site d'information permet aussi de répondre à des questions (sous forme de FAQ) liées à l'utilisation et à l'évolution du dispositif, et donne accès aux résultats issus des exploitations de la base nationale, aux niveaux national et régional. Il met enfin à disposition des réflexions et expériences autour de différents usages d'Evrest et plus largement des chiffres en santé au travail.

Ces éléments «techniques » permettent donc de fournir à chaque médecin des outils lui facilitant un usage personnalisé d'Evrest, tout en constituant un lieu d'échange d'informations et d'expériences, et en mettant à la disposition de tous des résultats par secteurs, métiers, etc. (sous réserve d'effectifs suffisants) permettant de choisir d'éventuelles « références » pour des comparaisons aux résultats locaux.

\subsection{Un mode d'organisation original : des formes diverses de partenariat et de réseaux, à différents niveaux}

Le dispositif se met progressivement en place depuis le $1^{\mathrm{er}}$ janvier 2007, date à laquelle il a démarré à titre expérimental dans une région française (le Nord Pas de Calais). Avec l'ouverture au niveau national à partir de 2008 du site d'information et d'un site pour l'inscription des médecins et la saisie, le dispositif a pu démarrer dans d'autres régions. Mais son développement n'est que très progressif, ne serait-ce que parce la possibilité de s'inscrire dans Evrest dépend de l'existence d'un début de structuration au niveau régional, structuration qui ne se met en place qu'à l'initiative de médecins de la région et pas de l'équipe nationale (cf. ci-après).

Au $1^{\text {er }}$ juillet 2011, 1229 médecins du travail s'étaient inscrits dans Evrest (mais tous n'ont pas saisi de fiches de salariés). La base nationale (salariés nés en octobre d'une année paire) était constituée de près de 10800 fiches pour 2008, plus de 13900 pour 2009, et près de 12300 pour 2010.

Tableau 1

\begin{tabular}{|l|l|l|l|l|l|}
\hline Nombre de fiches* saisies & $\begin{array}{l}2007 \\
\text { (expérimentation) }\end{array}$ & $\mathbf{2 0 0 8}$ & $\mathbf{2 0 0 9}$ & $\mathbf{2 0 1 0}$ & TOTAL \\
\hline
\end{tabular}




\begin{tabular}{|l|l|l|l|l|l|}
\hline $\begin{array}{l}\text { Fiches échantillon national (octobre } \\
\text { années paires) }\end{array}$ & $\mathbf{2 5 0 3}$ & $\mathbf{1 0} \mathbf{8 0 5}$ & $\mathbf{1 3 9 1 4}$ & $\mathbf{1 2} \mathbf{2 7 7}$ & $\mathbf{3 9} \mathbf{4 9 9}$ \\
\hline $\begin{array}{l}\text { Fiches hors échantillon national (liées à } \\
\text { des usages spécifiques) }\end{array}$ & 4806 & 15172 & 18169 & 22250 & 60397 \\
\hline Ensemble des fiches saisies & 7309 & 25977 & 32083 & 34527 & 99896 \\
\hline
\end{tabular}

* Une fiche correspond à un questionnaire pour un salarié lors d'une visite ayant donné lieu à Evrest

47 Le dispositif se caractérise par trois grands niveaux de fonctionnement: le niveau national, le niveau régional, et les médecins participants. À chacun de ces niveaux se nouent des formes plurielles d'échanges et de partenariat autour d'Evrest qui mériteraient d'être analysées plus en profondeur que nous ne pouvons le faire nousmêmes.

Le niveau national est, depuis 2009, structuré autour d'un groupement d'intérêt scientifique (GIS Evrest), associant plusieurs organismes publics ou de recherche s'intéressant à l'amélioration des conditions de travail et à la santé au travail, l'organisme coordonnant les services interentreprises de médecine du travail, et l'entreprise aéronautique dans laquelle Evrest a pris naissance ${ }^{8}$. La création de ce GIS stabilise l'existence du dispositif, en lui donnant une meilleure visibilité institutionnelle, en lui assurant des financements pour fonctionner et en confortant ses objectifs scientifiques. Un conseil scientifique, constitué de six personnalités de disciplines différentes ${ }^{9}$, apporte son éclairage critique et un appui dans les réflexions. Ce GIS matérialise aussi le besoin ressenti, pour faire exister le dispositif, de le faire reconnaître par différents "alliés » parmi des partenaires publics et de recherche, dont les intérêts peuvent rejoindre ce qui se joue dans le dispositif Evrest. On peut sur ce point encore rapprocher la démarche de celle décrite par Star et Griesemer à propos des alliances qui se sont faites autour de la création du musée de zoologie et des objets-frontière qui deviennent les supports de ces rencontres entre des univers professionnels.

Le fonctionnement du niveau national repose principalement sur la directrice du GIS (coauteure du présent article) et sur les membres d'une équipe projet (Équipe Projet Nationale Evrest) qui rassemble des médecins du travail, des chercheurs en santé au travail et des statisticiens. Ils sont chargés de la mise en œuvre du dispositif, de son évolution, et d'alimenter et animer la réflexion de l'ensemble des médecins participants autour des usages des chiffres en santé au travail. Ils sont aussi responsables de la constitution, de la gestion et de l'exploitation de l'échantillon national. Ils ont en charge l'animation du réseau des médecins référents régionaux, qui font le lien entre les trois niveaux de fonctionnement d'Evrest. Cette équipe projet, encore très largement composée de ceux qui ont construit le dispositif Evrest, est le lieu central où se tissent les compromis, où se négocient les évolutions du dispositif, où se préparent les discussions du réseau et des instances de direction du GIS. La plupart des médecins qui y participent sont aussi des référents pour leur région.

50 Car le dispositif Evrest a un ancrage régional fort. En effet, il ne fonctionne qu'avec des médecins référents régionaux volontaires, qui permettent à des médecins de la région de participer, puisque toute inscription d'un médecin souhaitant participer à Evrest suppose un échange et une validation par le médecin référent de sa région. En l'absence de 
médecin référent, aucun médecin de la région ne peut s'inscrire - donc avoir ses log in et mot de passe qui l'autorisent à accéder au site de saisie. En 2011, quatre régions françaises n'ont pas de référents et les régions dans lesquelles le dispositif s'est le plus développé sont encore celles dans lesquelles les médecins référents ont fait partie de l'équipe qui a initialement porté le projet au niveau national. Chaque région a une indépendance de fonctionnement et de gestion, et le médecin référent doit aussi contribuer à constituer, faire vivre et structurer le réseau de sa région, afin d'assurer le financement et la logistique du projet à ce niveau. Cette activité du médecin référent régional est plus ou moins facilitée en fonction du service dans lequel il travaille, du caractère plus ou moins structuré des acteurs régionaux de la santé au travail et de leur positionnement plus ou moins favorable à Evrest. La constitution du réseau et sa consolidation au niveau régional prennent du temps, et doivent souvent surmonter des obstacles multiples. D'où un développement d'Evrest très inégal selon les régions. Le réseau, dans certaines régions, s'est ouvert à une collaboration avec d'autres partenaires institutionnels (observatoires régionaux de santé, directions régionales du travail, Institut de médecine du travail...). En fonction des particularités régionales qui peuvent être liées à l'insertion professionnelle du médecin référent, à l'histoire des régions, mais aussi à des personnalités du côté des « institutionnels » dans le champ de la santé au travail (universitaires en santé au travail, inspection médicale, etc.), les alliances trouvées autour d'Evrest et les réseaux d'acteurs impliqués ne sont pas les mêmes. Les modes d'intégration d'Evrest aux préoccupations des acteurs régionaux de la santé au travail peuvent donc différer sensiblement. Toutes ces questions émergent lors des réunions des médecins référents régionaux, qui se retrouvent deux fois par an, avec l'équipe projet, afin d'échanger sur leurs expériences, leurs difficultés, et sur les usages d'Evrest.

51 Le travail en réseau est une autre spécificité d'Evrest, et pas seulement avec les médecins référents régionaux. Dans plusieurs régions, des référents statistiques Evrest ont été peu à peu adjoints pour le traitement des données régionales. En lien avec le médecin référent, ils apportent aussi leur concours aux médecins du travail qui se lancent dans des usages spécifiques d'Evrest (en entreprise). Ces référents statistiques ont souhaité tisser des liens entre eux, et ils se retrouvent (au moins deux fois par an) pour échanger sur leurs expériences, repérer les ressources liées aux compétences de chacun d'eux, et développer une culture commune, sur Evrest et plus généralement sur les approches en santé au travail.

D'autres réseaux se créent de façon plus ponctuelle autour d'Evrest. Ainsi, des médecins intéressés se sont associés pour un travail en commun sur des professions telles que les aides à domicile ou les ripeurs. Dans une région, quelques médecins se sont retrouvés entre pairs pour échanger autour de présentations de résultats issus d'investigations spécifiques dans des entreprises, et de la façon d'y intégrer leurs connaissances qualitatives. Il est possible que l'introduction des questions « libres » suscite le besoin de trouver des formes d'échanges et de coordination entre des médecins souhaitant approfondir des thématiques particulières. Il est trop tôt pour le dire, mais il semble bien que l'usage d'Evrest et le mode de structuration du dispositif favorise jusqu'à présent, au moins pour une partie des médecins participants, des modalités d'échange qui se situent un peu en dehors des cadres habituels de la profession, et qui portent à la fois sur leur usage d'Evrest et sur son intégration dans leur pratique professionnelle. 


\section{Un dispositif en tension} dispositif toujours en tension. Sa validité ne sera reconnue dans le champ scientifique que s'il a un apport original et de qualité dans la production de connaissances en santé au travail, mais son existence et sa pérennité reposent sur la participation de médecins de terrain volontaires, qui doivent y trouver un intérêt durable pour leur propre pratique. Cette caractéristique d'objet-frontière est ce qui fait son originalité et sa richesse, mais aussi ce qui le rend fragile.

\subsection{Un échantillon représentatif?} seulement d'un choix méthodologique lié aux doutes qu'on pourrait avoir sur la qualité des données recueillies et sur la possibilité d'inscrire le dispositif dans la durée si un médecin n'était pas intéressé par Evrest. La démarche de développement du dispositif, dans la mesure où elle vise une participation active des médecins, n'a pas été de faire en sorte que le maximum de médecins s'inscrive coûte que coûte, mais de construire peu à peu des réseaux avec ceux qui le souhaitent, car les médecins sont des acteurs du dispositif, ceux qui contribuent à le construire et à le faire vivre (notamment dans les régions), mais aussi ceux qui peuvent « donner un sens aux données » par leur expérience clinique. Ces choix engendrent cependant des difficultés, en particulier (pour éviter la répétition de «notamment»)pour assurer la représentativité de l'échantillon: pour s'inscrire dans une région, il faut préalablement une amorce de réseau et un médecin volontaire pour devenir référent ; la participation des médecins au sein d'une région peut varier selon les services et la plus ou moins grande proximité, intellectuelle ou géographique, avec le médecin référent; la participation des médecins des services autonomes ou des fonctions publiques est moins importante que celle des médecins des services interentreprises de santé au travail ${ }^{10}$, etc. Ces difficultés ont un impact plus fort encore dans la perspective du suivi longitudinal (nous y reviendrons).

Malgré ces limites, compte tenu du grand nombre de médecins qui se sont inscrits jusqu'à présent dans le dispositif, l'échantillon national Evrest est très important (voir tableau supra). Ainsi, pour les années 2008-2009, l'exploitation pouvait porter sur 22298 salariés. Les caractéristiques de cet échantillon ont été comparées, sur quelques variables socioprofessionnelles, à d'autres échantillons nationaux, et globalement, l'échantillon Evrest apparaît assez cohérent avec ces sources. Il faut cependant rester prudent dans la lecture des résultats descriptifs, notamment s'il s'agit d'apprécier des niveaux (de telle ou telle caractéristique professionnelle ou de santé). La non-représentativité de l'échantillon est accentuée au niveau régional et la comparaison de résultats régionaux entre eux est difficilement envisageable du fait des différences de structure des populations de salariés enquêtés, résultant à la fois de la diversité des structures socioéconomiques régionales et des participations inégales des services à l'intérieur de chaque région. L'utilisation d'Evrest pour comparer des groupes professionnels (catégories socioprofessionnelles, secteurs d'activité, etc.) semble en revanche un peu moins sensible aux problèmes de

Perspectives interdisciplinaires sur le travail et la santé, 13-2 | 2011 
représentativité et la taille potentiellement importante de la base Evrest en fait une des rares sources permettant des investigations sur les relations entre le travail et la santé dans un grand nombre de sous-populations ciblées. Des travaux ont d'ores et déjà été amorcés dans cette perspective ${ }^{11}$.

Encadré 1. Quelques informations sur la médecine du travail en France

L'organisation de la médecine du travail est en France régie par le code du travail. Elle s'applique à tous les salariés. Les médecins du travail exercent leur activité soit dans des services autonomes au sein de grandes entreprises, soit dans des services interentreprises. Les médecins du travail ont un rôle exclusivement préventif en matière de risques professionnels, en dehors des premiers secours. Leurs actions se partagent entre des actions de prévention sur le milieu de travail et les visites médicales des salariés. Ils sont censés informer et conseiller l'employeur sur les risques liés à l'activité de travail et sur la santé collective des salariés. Ils participent aux débats sur la santé et la sécurité des salariés, notamment dans les comités d'hygiène, de sécurité et des conditions de travail.

On distingue plusieurs types d'entretiens médicaux, notamment : des entretiens d'embauche ; des entretiens dits périodiques ou systématiques (programmés tous les deux ans maximum) - pour certaines expositions ou situations de travail, une surveillance médicale renforcée est prévue par les textes; et, en cas de problème de santé, des entretiens médicaux à la demande (du salarié, de l'employeur ou du médecin) et des visites de reprise du travail (lorsqu'un salarié a eu un arrêt de travail d'une durée définie par la loi, variable selon le contexte). Les textes prévoient que les entretiens médicaux donnent lieu à l'établissement d'un certificat médical d'aptitude au poste de travail.

Du fait de la pyramide des âges des médecins du travail, leur nombre est en forte diminution depuis quelques années (ils sont environ 5700 encore en activité à ce jour), et cette tendance devrait s'accentuer dans les années à venir. Ce déficit de médecins a amené des réflexions sur une réforme en cours, prévoyant notamment une large place aux infirmières de santé au travail dans les équipes pluridisciplinaires coordonnées par les médecins du travail.

Deuxième obstacle, très dépendant du contexte actuel de pénurie de médecins du travail et d'incertitude sur le cadre juridique de fonctionnement des services de santé au travail (voir encadré), celui d'avoir un échantillonnage qui ne sur- ou sous- représente pas les problèmes de santé. Cette question est très liée au mode d'organisation des visites et aux occasions que les salariés vont avoir de rencontrer le médecin du travail. A priori, tous les salariés suivis par la médecine du travail sont susceptibles d'entrer dans Evrest, s'ils ont un emploi d'une durée d'au moins deux mois (y compris les intérimaires). Et, pour éviter de surreprésenter ceux ayant des troubles de santé ou des salariés qui sont là depuis trop peu de temps, le questionnaire devrait être passé lors des entretiens systématiques de santé au travail (que chaque salarié devrait avoir, quel que soit son état de santé et ses expositions professionnelles, soit tous les ans, soit tous les deux ans) - voire lors de certains entretiens d'embauche si ceux-ci ont lieu alors que le salarié est en poste depuis au moins deux mois, ou encore lors de certaines visites de reprise après un arrêt de travail court. Interroger les salariés lors de la visite systématique peut cependant être 
source de biais dans plusieurs sens : inévitablement, celui de surreprésenter les salariés en bonne santé, car seuls ceux qui ne sont pas en arrêt de travail long peuvent participer, mais aussi, comme les médecins du travail ont de moins en moins de temps à consacrer aux visites systématiques, celui de surreprésenter les salariés ayant des conditions de travail difficiles s'ils bénéficient de ce fait d'une «surveillance médicale renforcée » et sont donc susceptibles d'être vus plus souvent en visite systématique. Pour remédier en partie à ces difficultés, les exploitations nationales d'Evrest portent sur les fiches de deux années consécutives (seule la visite la plus récente est exploitée pour les salariés vus deux fois sur la période). Mais, en fonction des évolutions du cadre réglementaire des services de santé au travail (en pleine réforme à l'heure où cet article est rédigé), il est probable que des coopérations pour certaines tâches au sein des services (avec notamment un rôle accru des personnels infirmiers) pourraient conduire à revoir les modes d'organisation des visites systématiques, et donc les conditions de passation du questionnaire Evrest. Avec probablement des possibilités d'améliorer la qualité de l'échantillonnage, mais aussi avec des questions nouvelles à mettre en discussion, s'il y a une participation plus active d'autres acteurs que le médecin du travail dans le recueil des données.

\subsection{La gageure du longitudinal}

58 Le dispositif Evrest a été construit comme un "observatoire», c'est-à-dire pour permettre d'assurer à la fois des « photographies » répétées dans le temps du travail et de la santé, mais aussi un suivi du travail et de la santé des salariés. Les données longitudinales sont en effet nécessaires à l'étude des liens entre les conditions de travail et la santé, permettant des explorations diachroniques (Molinié et Pueyo, à paraître).

59 Techniquement, le suivi longitudinal est assuré dans le respect de l'anonymat grâce à un algorithme de hachage, qui crée un identifiant unique par salarié à partir d'informations d'identification stables dans le temps. L'utilisation de cet identifiant permet un suivi des salariés, indépendant de leur lieu de travail (entreprise, région...). Ceci suppose néanmoins la ré-interrogation régulière par des médecins du travail participants. $\mathrm{Ne}$ pourront donc être suivis que les salariés qui seront en emploi, dont le médecin du travail participe à Evrest, qui seront vus lors d'une visite périodique (ou assimilée), et qui y viendront. Or l'instabilité de l'emploi, l'augmentation de la précarité et le turn-over accru diminuent la probabilité qu'un salarié soit vu de façon régulière lors d'une visite systématique. Par ailleurs, l'évolution du contexte réglementaire et la démographie des médecins du travail conduisent à un espacement des entretiens médicaux. À ce jour, le recul est encore insuffisant pour nous permettre de chiffrer la fraction de salariés qui pourra être suivie, mais on sait déjà que la part des visites systématiques devient de plus en plus faible dans l'activité de nombreux médecins du travail. Et il n'est pas encore possible de donner de premiers résultats longitudinaux puisque les analyses ne portent que sur des groupes de deux années consécutives et que le dispositif n'a démarré progressivement au niveau national qu'en $2008^{12}$.

60 La réflexion sur ce sujet devra être poursuivie pour ancrer le dispositif Evrest dans la durée, en lien avec les évolutions réglementaires dans le champ de la santé au travail, tout en conservant un rôle essentiel aux médecins du travail.

61 De plus, la participation des médecins du travail reposant sur le volontariat, il est important que leur intérêt pour Evrest se maintienne au fil du temps, ce qui sera probablement d'autant plus le cas qu'ils développeront des usages spécifiques du 
dispositif, seuls ou de façon coordonnée avec des collègues. Mais il est aussi essentiel pour le dispositif qu'ils comprennent l'intérêt (au-delà de l'engagement formel qu'ils prennent au moment de leur inscription) de contribuer à la constitution de la base nationale, ne serait-ce que pour situer leurs propres résultats dans un ensemble plus vaste (secteurs, métier, etc.), mais aussi pour permettre des travaux scientifiques contribuant, à un autre niveau, aux réflexions sur la santé au travail. Or, il est sûr que ne faire durablement Evrest que pour les seuls salariés de l'échantillon national - donc presque toujours insuffisamment nombreux pour permettre de produire des résultats locaux pertinents est peu incitatif pour qu'un médecin continue à participer au dispositif (malgré son engagement initial) ; à l'inverse, l'intérêt suscité par des usages spécifiques peut conduire à «oublier » d'interroger les salariés de l'échantillon national, s'ils ne sont pas dans l'entreprise ou le secteur ciblé pour ces investigations particulières. Par construction, l'échantillon et son suivi longitudinal sont techniquement problématiques, puisqu'ils dépendent étroitement du taux de couverture du dispositif, de l'engagement durable des médecins participants, et de la possibilité, à l'intérieur de l'organisation de la médecine du travail, d'assurer un suivi régulier des salariés le plus indépendant possible de leurs "expositions " ou de leur état de santé. Sur ce point encore, on voit bien que la possibilité même de survie du dispositif, et donc aussi du suivi longitudinal, ne peut être pensée seulement de manière « technique ». Elle est au cœur de la tension, inhérente au dispositif, entre les usages à différents niveaux et par différents professionnels. Avancer sur ce point supposera certes de bien inscrire toute production de résultats dans les caractéristiques et les limites du dispositif, mais aussi d'accorder une très grande attention aux activités d'articulation, de construction et d'animation des divers réseaux, pour que les préoccupations issues de l'activité de chacun des acteurs concernés et la diversité des points de vue puissent s'y exprimer et être mises en débat. C'est sans doute à cette condition qu'Evrest pourra continuer à être à la fois un dispositif pour la production de connaissances à un niveau national, connaissances enrichies de la participation active de ceux qui les produisent, et un dispositif à usage local pour contribuer à la visibilité des enjeux de travail et de santé dans les entreprises, tout en ayant l'appui d'un réseau de réflexion et des possibilités de comparaison qui dépassent ce cadre local.

\section{BIBLIOGRAPHIE}

Archambault C., Doppler F., Tessoulin C., Stocker M.M., Beaugrand M., Jacquet C., Niezborala M., Tramier A., Sénécal S., Darbon L., Molinié A.-F. (2006). Observatoire Evrest, outil de veille et de suivi de la santé des salariés au travail dans le secteur aéronautique. Archives des maladies professionnelles et de l'environnement, vol. 67, $\mathrm{n}^{\circ} 2,333$.

Arnaudo B., Magaud-Camus I., Sandret N., Coutrot T., Floury M.-C., Guignon N., Hamon-Cholet S., Waltisperger D. (2004). L'exposition aux risques et aux pénibilités du travail de 1994 à 2003.

Premiers résultats de l'enquête SUMER 2003. Premières synthèses, DARES, décembre 2004, n 52.1, 
8 p. www.travail-emploi-sante.gouv.fr/IMG/pdf/publication_pips_200412_n-52-1_expositionrisques-penibilites-travail.pdf

Askenazy P., Cartron D., de Coninck F., Gollac M. (coord.). (2006). Organisation et intensité du travail. Toulouse: Éditions Octarès.

Balogh I., Ørbæk P., Ohlsson K., Nordander C., Unge J., Winkel J., Hansson G.-A., the Malmö Shoulder/Neck Study Group. (2004). Self-assessed and directly measured occupational physical activities - influence of musculoskeletal complaints, age and gender. Applied Ergonomics, vol. 35, 49-56.

Bowker G., Star S.L. (1999). Sorting Things Out: Classification and its Consequences. Cambridge, MA : MIT Press.

Bué J., Coutrot T., Hamon-Cholet S., Vinck L. (2007). Conditions de travail : une pause dans l'intensification du travail. Premières synthèses, DARES, janvier 2007, nº 01.2, 7 p. www.travailsolidarite.gouv.fr/IMG/pdf/2007.01-01.2.pdf

Célérier S. (2008a). Cancer et activités professionnelles des malades : les enseignements de trente ans de littérature internationale sur le thème. Sociologie et santé, nº 28, juin, 185-199.

Célérier S. (2008b). Santé précaire au travail : quelques perspectives sociologiques. Connaissance de l'emploi, CEE, n56, 1-4. www.cee-recherche.fr/fr/connaissance_emploi/56sante_precaire_travail_sociologie.pdf

Chassaing K., Waser A.-M. (2010). Travailler autrement. Comment le cancer initie un autre rapport au travail ? Travailler, $\mathrm{n}^{\circ} 23,99-126$.

Collège d'expertise sur le suivi statistique des risques psychosociaux au travail. (2009). Indicateurs provisoires de facteurs de risques psychosociaux au travail. Octobre 2009. www.collegerisquespsychosociaux-travail.fr/site/medias/Indicateurs-provisoires.pdf

Coutrot T., Molinié A.F. (2006). Des grandes enquêtes vers les indicateurs de santé en entreprise : les « grandes enquêtes » et les médecins du travail. Archives des maladies professionnelles et de l'environnement, vol. 67, 2, 323-327.

Dejours C. (1993). Travail : usure mentale, de la psychopathologie à la psychodynamique du travail. Paris : Éditions Bayard.

Derriennic F., Touranchet A., Volkoff S. (1996). Estev, une méthode d'enquête. In F. Derriennic, A. Touranchet, S. Volkoff (coord). Âge, travail, santé. Études sur les salariés âgés de 37 à 52 ans. Enquête Estev 1990. Les Éditions Inserm, (collection Questions de santé publique). 33-54.

Desrosières A. (1993). La politique des grands nombres. Histoire de la raison statistique. Paris : La Découverte (Textes à l'appui).

Desrosières A. (2004). Enquêtes versus registres administratifs : réflexions sur la dualité des sources statistiques. Courrier des statistiques, $\mathrm{n}^{\circ} 111$, septembre 2004, 3-15. www.insee.fr/fr/ffc/ docs_ffc/cs111b.pdf

Doppler F. (1995). Évolution de la population et transformation ou conception des situations de travail. In J.C. Marquié, D. Paumès et S. Volkoff (coord.). Le travail au fil de l'âge. Toulouse : Éditions Octarès. 411- 427.

Doppler F. (2004). Travail et santé. In Falzon P. (coord.). Ergonomie. Paris : PUF. 69-82.

Duquette J., Lortie M., Rossignol M. (1997). Perception of difficulties for the back related to assembly work : general findings and impact of back health. Applied Ergonomics, vol. 28, $\mathrm{n}^{\circ} \mathrm{s} 5-6$, 389-396. 
Falzon P., Doppler F. (1998). 10 ans après : l'évaluation à long terme d'une action ergonomique. XXXIII ${ }^{e}$ Congrès de la SELF. Paris, 16-17-18 septembre 1998. http://ergonomie.cnam.fr/equipe/ falzon/articles_pf/10\%20ans\%20apres_98.pdf

Fougeyrollas P. (2002). L'évolution conceptuelle internationale dans le champ du handicap : enjeux socio-politiques et contributions québécoises. Pistes, vol. 4, n², novembre 2002. www.pistes.uqam.ca/v4n2/articles/v4n2a12.htm

Gilles M. (2006). Genèse et usages d'un outil quantitatif en santé au travail dans une entreprise. Intérêts, limites et obstacles d'une démarche d'objectivation des liens entre santé et travail. Mémoire pour le master « Organisation, ressources humaines communication et emploi ». Spécialité Organisation de la production et de l'entreprise. Université de Marne la Vallée. En ligne sur le site Evrest : evrest.istnf.fr/_admin/Repertoire/fckeditor/file/Docs_PDF/

MemoireMasterMGilles.pdf

Gilles M., Volkoff S. (2009). Quantifier la santé au travail en entreprise : acteurs, conventions, usages. Article de synthèse à la suite de la convention ANR n 05954/ANR 05 SEST 032-01. Disponible sur le site de l'ANR www.agence-nationale-recherche.fr/documents/uploaded/2009/ SEST2005/28wolkoff.pdf

Gollac M. (1997). Des chiffres insensés ? Pourquoi et comment on donne un sens aux données statistiques. Revue française de sociologie, $\mathrm{n}^{\circ}$ XXXVIII-1, 1-36.

Gollac M., Volkoff S. (2007). Les conditions de travail. Paris, Éditions La Découverte (Collection Repères), $\mathrm{n}^{\circ} 301$.

Laville A. (1998). Les silences de l'ergonomie vis-à-vis de la santé. In Actes du colloque « Recherche et ergonomie » - Toulouse 1998. 151-158. http://www.ergonomie-self.org/media/media30419.pdf

Lhuilier D., Amado S., Brugeilles F., Diallo M., Rolland D. (2010). Le travail et l'emploi à l'épreuve du VIH/VHC : régulations des systèmes d'activités et réévaluation du modèle de vie. @ctivités, vol. 7 (2), 2-26. www.activites.org/v7n2/v7n2.pdf

Millanvoye M., Colombel J. (1996). Âge et activité des opérateurs dans une entreprise de construction aéronautique. In R. Patesson (dir.) Intervenir par l'ergonomie, XXXI ${ }^{\mathrm{e}}$ Congrès de la SELF, Bruxelles. vol. 2, 39-46.

Minaire P. (1992). Disease, Illness and Health : The critical Models of the Disablement Process, Bulletin of the World Health Organization, vol. 70 (3), 373-379.

Molinié A.-F., Volkoff S. (2000). Intensité du travail et santé dans un organisme administratif : une enquête à l'Agence nationale pour l'emploi. PISTES, vol. 2, n 1, mai 2000.

Molinié A.-F. (2003). Interroger les salariés sur leur passé professionnel : le sens des discordances. Revue d'épidémiologie et de santé publique, nº 51, p. 589-605.

Molinié A.-F. (2006a). La santé au travail des salariés de plus de 50 ans. In Insee, Données sociales, La Société Française. Édition 2006. 543-553. www.insee.fr/fr/ffc/docs_ffc/donsoc06zi.pdf

Molinié A.-F. (2006b). Les salariés quinquagénaires, entre fragilisation et protection. Retraite et société, $\mathrm{n}^{\circ} 49,12-37$.

Molinié A.-F., Pueyo V. (à paraître). Les dynamiques temporelles des relations santé travail, et le fil de l'âge. In Molinié, Gaudart, Pueyo (coord.). La vie professionnelle : âge, expérience et santé à l'épreuve des conditions de travail. Toulouse : Éditions Octarès (Travail et activité humaine).

Molinié A.-F., Volkoff S. (1982). Quantifier les conditions de travail ? Travail et Emploi, $\mathrm{n}^{\circ}$ 11, p. 63-70. 
Nagi S.Z. (1964). A study in the evaluation of disability and rehabilitation. Potential concepts, methods, and procedures. American Journal of Public Health, vol. 54, n 9, 1568-1579.

Star S.L. (2010). Ceci n'est pas un objet-frontière ! Réflexions sur l'origine d'un concept. Revue d'anthropologie des connaissances, 2010/1, vol. 4, n 1, 18-35.

Star S.L., Griesemer J.R. (1989). Institutional Ecology, 'Translations' and Boundary Objects : Amateurs and Professionals in Berkeley's Museum of Vertebrate Zoology, 1907-1939. Social Studies of Science, vol. 19, $n^{\circ} 3,387-420$.

Teiger C., Laville A. (1991). L'apprentissage de l'analyse ergonomique du travail, outil d'une formation pour l'action. Travail et Emploi, $\mathrm{n}^{\circ} 47,10$ p. http://ecrire.travail-solidarite.gouv.fr/ publications/Revue_Travail-et-Emploi/pdf/47_3091.pdf

Trompette P., Vinck D. (2009). Retour sur la notion d'objet-frontière. Revue d'anthropologie des connaissances, 2009/1, vol. 3, $\mathrm{n}^{\circ} 1,15-27$.

Trompette P., Vinck D. (2010). Retour sur la notion d'objet-frontière (2). Fécondité de la notion dans l'analyse écologique des objets innovants. Revue d'anthropologie des connaissances, 2010/1, vol. $4, \mathrm{n}^{\circ} 1,11-15$.

Volkoff S. (2008). L'intensité du travail « disperse » les problèmes de santé. In de Terssac G., Saint-Martin C. et Thébault C. (coord.). La précarité : une relation entre travail, organisation et santé. Toulouse : Éditions Octarès. 29-40.

Volkoff S. (coord.) 2005. L'ergonomie et les chiffres de la santé au travail : ressources, tensions et pièges. Toulouse : Éditions Octarès.

Volkoff S., Molinié A.-F. (2010). Quantifier sans broyer ? Les statistiques en santé au travail à la rencontre des analyses cliniques. In D. Lhuilier et Y. Clot (coord.). Travail et santé- Ouvertures cliniques. Toulouse : Éditions Erès (Clinique du travail). 175-188.

Wood P.H.N. (1975). Classification of Impairments and Handicaps, World Health Organization, (WHO/ ICD 9/REV. CONF/75, 15).

World Health Organization (WHO) (1980). International Classification of Impairments, Disabilities, and Handicaps, Geneva : World Health Organization (Reprint 1993). 


\section{ANNEXES}
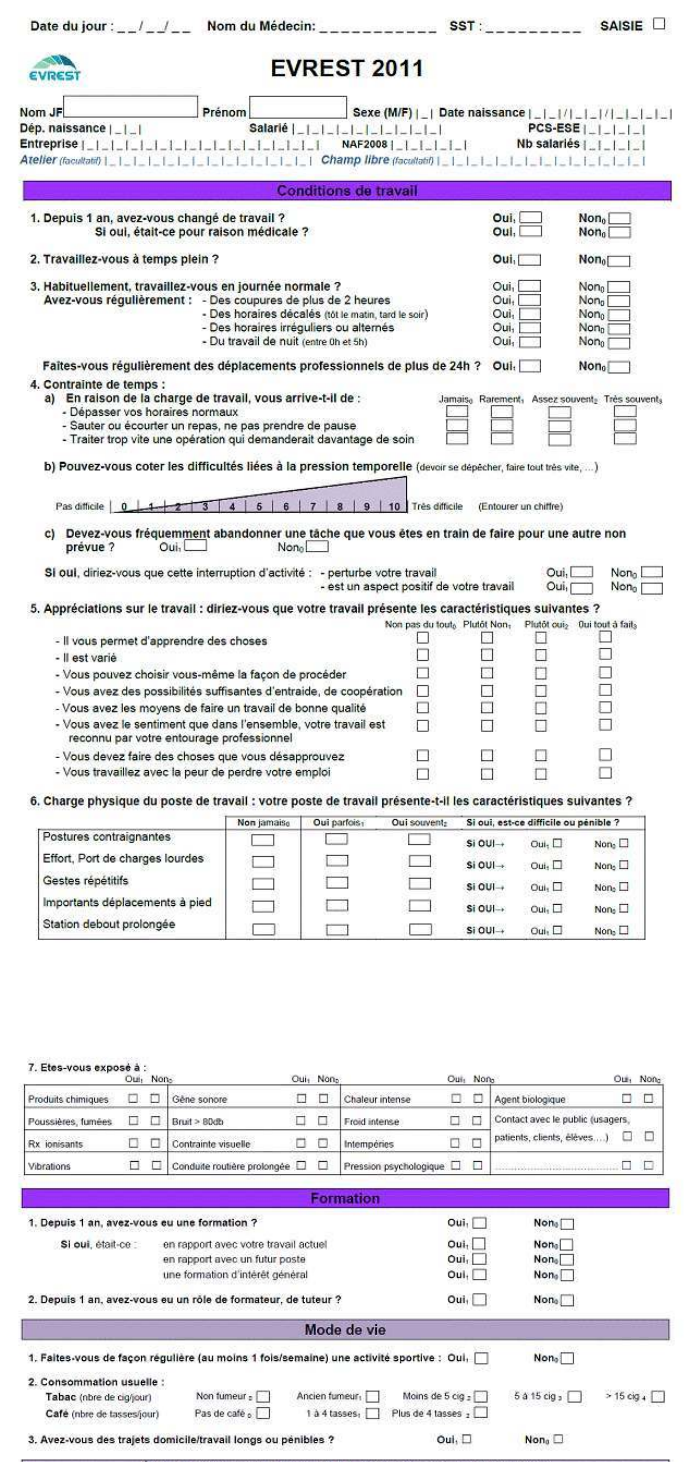

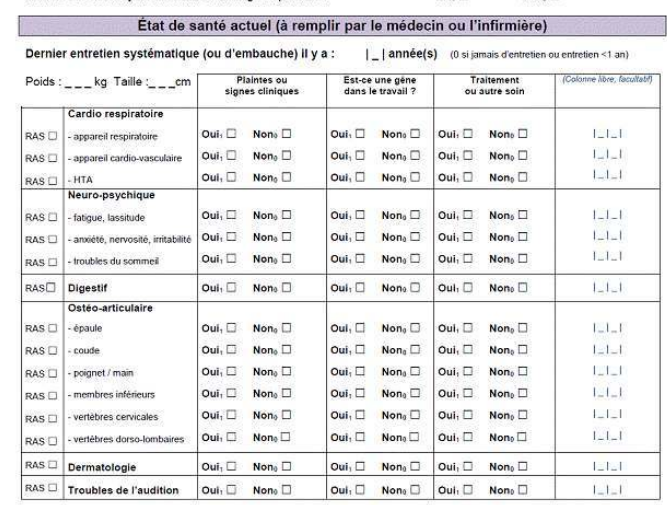




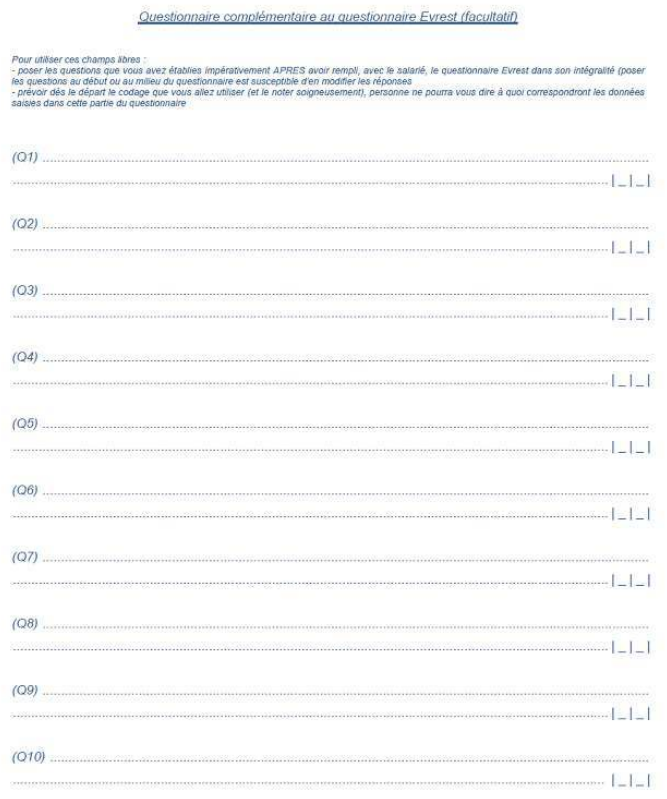

\section{NOTES}

1. «Un objet est quelque chose sur et avec lequel des personnes (ou en informatique, d'autres objets ou logiciels) agissent. Sa matérialité provient de l'action et non d'un sens préfabriqué de la matière ou de sa qualité de chose. » (Star, 2010, p. 20).

2. Marion Gilles (Modys, Université Lyon 2), thèse de Sociologie en cours sous la direction de Michel Gollac. Sujet de thèse : Fabrications et usages sociaux des chiffres en santé-travail en entreprises. Enjeux et tensions autour de la représentation de la « Santé au travail ».

3. Centre Interservices de Santé et de Médecine du travail en Entreprise. Cet organisme coordonne les services interentreprises de santé au travail.

4. Un « collège d'expertise » a reçu pour mission de formuler des propositions en vue d'un suivi statistique des risques psychosociaux au travail. Dans un premier rapport remis en octobre 2009 au Ministre du travail, il a proposé une quarantaine d'indicateurs immédiatement disponibles dans les sources statistiques françaises existantes. Ces indicateurs reflètent six dimensions retenues pour analyser ces risques : 1 / les exigences du travail ; 2 / les exigences émotionnelles ; 3/ l'autonomie et les marges de manœuvre ; 4/ la qualité des relations au travail ; 5/ les conflits de valeurs (problèmes d'éthique et de conscience professionnelle) ; 6/ l'insécurité de l'emploi et de la carrière. Voir http://www.college-risquespsychosociaux-travail.fr/index.cfm

5. Voir notamment des recherches sur le travail à l'épreuve du cancer ou du VIH/VHC (Chassaing et Waser, 2010 ; Lhuilier et coll., 2010 ; Célérier, 2008a et b).

6. On en trouvera quelques exemples dans la Lettre Evrest qui est disponible sur le site http:// evrest.istnf.fr

7. La base de données est alimentée par toutes les fiches saisies sur le site par les médecins participants. Elle est hébergée sur un serveur sécurisé, et a fait l'objet d'une autorisation de mise en œuvre par la Commission nationale informatique et libertés (CNIL). Les droits d'accès font 
également l'objet d'une charte d'utilisation des données que tout participant au dispositif s'engage à respecter.

8. Les partenaires du GIS Evrest sont: Agence Nationale pour l'Amélioration des Conditions de Travail (ANACT) ; Agence nationale de sécurité sanitaire de l'alimentation, de l'environnement et du travail (ANSES); Centre de recherches et d'études sur l'âge et les populations au travail (CREAPT) ; Institut de Santé au Travail du Nord de la France (ISTNF) ; Université Lille 2 (Droit et santé); Centre Interservices de Santé et de Médecine du travail en Entreprises (CISME); European Aeronautic Defence and Space company (EADS).

9. Président du conseil scientifique du GIS Evrest: Michel Gollac, directeur du laboratoire de sociologie quantitative CREST-INSEE. Les autres membres du conseil scientifique sont: Ellen Imbernon, directrice du Département santé travail de l'Institut de veille sanitaire; Philippe Mairiaux, professeur à l'Université catholique de Liège (Belgique), Département des sciences de la santé publique; Christophe Paris, professeur de médecine du travail, Nancy; Valérie Pueyo, maître de conférences en ergonomie à l'Université Lyon 2; Hélène Sultan-Taïeb, maître de conférences en économie à l'Université de Bourgogne, Dijon.

10. Cependant, quelques grandes entreprises utilisent déjà ou mettent en place un recueil de données très proche du dispositif national Evrest, mais indépendant dans son fonctionnement. Des échanges existent entre les médecins participant à ces divers dispositifs, notamment afin d'étudier des possibilités de rapprochement.

11. Ainsi, à partir de l'échantillon 2008-2009, des résultats comparatifs ont pu être présentés sur «Caractéristiques du travail, pression temporelle et gênes dans le travail liées à des lombalgies » pour deux populations ouvrières masculines : les ouvriers de «type industriel » travaillant dans le secteur de l'industrie ( $\mathrm{n}=1722)$ et les ouvriers de la construction $(\mathrm{n}=1505)$. (Communication d'A. Leroyer et A.-F. Molinié, au Congrès de l'Aderest, en septembre 2010 ; article en préparation). 12. Une première exploration des possibilités d'analyse est cependant en cours à partir d'un échantillon dit «cylindré " constitué des salariés interrogés au moins une fois au cours des années 2007 ou 2008, puis au moins une fois en 2009 ou 2010.

\section{RÉSUMÉS}

Le dispositif Evrest (Évolutions et RElations en Santé au Travail) est un observatoire pluriannuel par questionnaire, construit en collaboration par des médecins du travail et des chercheurs, pour pouvoir analyser et suivre différents aspects du travail et de la santé de salariés. Il vise à constituer une base nationale, sur un échantillon de salariés, pour des travaux à caractère scientifique, mais aussi à permettre à chaque médecin (ou à des médecins qui se coordonneraient) d'utiliser Evrest au-delà de l'échantillon national en fonction de ses préoccupations. Sa construction s'inscrit dans un processus visant à rendre compatibles des exigences propres à chacun de ces «mondes" professionnels. Elle passe par la création et le partage d'«objets», partage dans lequel chacun «retrouve son compte», mais aussi par l'élaboration progressive de formes nouvelles de coordination et d'échanges.

Evrest (EVolutions et RElations en Santé au Travail) is a monitoring system, with data collected year after year by a questionnaire that was developed by a team of occupational physicians and researchers working together, in order to monitor and analyze various aspects of work and employee health. A national database on a sample of employees is thus being created that can be 
used for scientific work, but it also allows each doctor (or team of doctors who wish to coordinate their work) to make use of Evrest beyond the national sample in relation to their own concerns. Its development is part of a process aiming to reconcile the diverse and specific requirements of both professional worlds. It involved designing and sharing "objects » in a way that would satisfy all the stakeholders. It also meant the gradual development of innovative ways for networking and exchanges.

El dispositivo Evrest (EVoluciones y RElaciones en Salud en el Trabajo) es un sistema de monitoreo, que año tras año recolecta datos, mediante un cuestionario elaborado con la colaboración de médicos laborales e investigadores, para poder analizar y seguir diferentes aspectos del trabajo y de la salud de los asalariados. Su objetivo es constituir una base de datos nacional, sobre una muestra de asalariados, para realizar trabajos de carácter científico ; pero también para permitir que cada médico (o grupo de médicos que coordinarían su trabajo) haga uso de Evrest mas allá de la muestra nacional en función de sus propias preocupaciones. Su construcción se inscribe dentro de un proceso dirigido a hacer compatibles las exigencias propias de cada uno de estos dos "mundos" profesionales. Implicó el crear y compartir "objetos", de una forma que fuera satisfactoria para todos los involucrados. También significó la elaboración progresiva de nuevas formas de coordinación y de intercambios.

\section{INDEX}

Mots-clés : santé au travail, conditions de travail, suivi longitudinal, médecins du travail, pluridisciplinarité, objet frontière

Palabras claves : salud laboral, condiciones de trabajo, seguimiento longitudinal, médicos laborales, pluridisciplinariedad, objeto fronterizo

Keywords : occupational health, working conditions, longitudinal design, occupational health doctors, multidisciplinarity, boundary object

\section{AUTEURS}

\section{ANNE-FRANÇOISE MOLINIÉ}

anne.molinie@cee-recherche.fr, CREAPT-CEE, 29, promenade Michel-Simon, 93166 NOISY LE GRAND Cedex, France

\section{ARIANE LEROYER}

ariane.leroyer@univ-lillle2.fr, UDSL, Département Universitaire de Santé au Travail, Faculté de Médecine, 59045 LILLE Cedex, France 